\title{
ANÁLISE DA INFLUÊNCIA DO NÚMERO DE CANDIDATOS A DOAÇÃO DE SANGUE NO LEAD TIME DE UM DOADOR EM UM HEMOCENTRO
}

\section{ANALYSIS OF THE INFLUENCE OF THE NUMBER OF BLOOD DONATION CANDIDATES ON A BLOOD DONOR'S LEAD TIME IN A BLOOD BANK}

\section{ANÁLISIS DE LA INFLUENCIA DEL NÚMERO DE CANDIDATOS A DONACIÓN DE SANGRE EN EL PLAZO DE PRUEBA DE UN DONANTE EN UN HEMOCENTRO}

\author{
Mateus Santana \\ Mestrando em Engenharia de Produção pela Universidade Federal de São Carlos (UFSCar) \\ mateus.95.santana@gmail.com \\ http://orcid.org/0000-0002-7406-2592
}

Beatriz Miwa Murase

Graduanda Engenharia de Produção, com ênfase em Software, pela Universidade Estadual de Maringá.

biamurase@gmail.com

https://orcid.org/0000-0001-5360-0462

Manoel Francisco Carreira

Doutorado em Engenharia de Produção pela Universidade Federal de Santa. Professor adjunto nivel A da Universidade Estadual de Maringá.

mfcarreira@uem.br

https://orcid.org/0000-0002-1039-8142

Gilberto Clóvis Antonelli

Doutorado em Engenharia Química pela Universidade Estadual de Maringá. Professor associado da Universidade Estadual de Maringá do Departamento de Engenharia de Produção

gcantonelli@uem.br

https://orcid.org/0000-0003-0083-0832

Editor Científico: José Edson Lara
Organização Comitê Científico
Double Blind Review pelo SEER/OJS
Recebido em 26.10.2020
Aprovado em 20.02.2021

Este trabalho foi licenciado com uma Licença Creative Commons - Atribuição - Não Comercial 3.0 Brasil 


\title{
Resumo
}

Objetivo: Determinar o lead time do processo de doação de sangue em um hemocentro e avaliar como ele varia com o aumento do número de doadores candidatos.

Metodologia: Os processos de doação de sangue foram mapeados utilizando técnicas de mapeamento de processos. Tais processos foram, então, convertidos em modelos de simulação de eventos discretos. As funções que descrevem cada processo foram calculadas via software Expertfit. Para identificação da correlação entre o lead time e o número de candidatos a doação, utilizou-se o método do coeficiente de correlação linear.

Originalidade/relevância: $O$ estudo avança na literatura de gestão de operações demonstrando a eficácia do método de modelagem e simulação de processos para avaliação e predição de impactos em processos no contexto de doação de sangue.

Principais resultados: Foi possível determinar o lead time médio do processo de doação de sangue, com $95 \%$ de nível de confiança e 5\% de erro. Em relação à correlação linear entre as variáveis, o coeficiente calculado resultou em 0,3 , indicando não haver correlação.

Contribuições teóricas/metodológicas: A pesquisa pode melhor evidenciar o uso de técnicas de modelagem e simulação de sistemas para gerenciamento de processos altamente estocásticos.

Palavras-chave: Hemocentro. Lead time. Simulação. Doação de sangue. Flexsim.

\begin{abstract}
Objective: To determine the lead time of the blood donation process in a blood center and to evaluate how it varies with the increase in the number of candidate donors.
\end{abstract}

Methodology: Blood donation processes were mapped using process mapping techniques. Such processes were then converted into discrete event simulation models. The functions that describe each process were calculated using Expertfit software. To identify the correlation between the lead time and the number of candidates for donation, the linear correlation coefficient method was used.

Originality / relevance: The study advances in the operations management literature demonstrating the effectiveness of the process modeling and simulation method for assessing and predicting impacts on processes in the context of blood donation.

Main results: It was possible to determine the average lead time of the blood donation process, with 95\% confidence level and 5\% error. Regarding the linear correlation between the variables, the calculated coefficient resulted in 0.3 , indicating that there is no correlation.

Theoretical / methodological contributions: The research can better demonstrate the use of modeling and system simulation techniques for managing highly stochastic processes.

Keywords: Blood center. Lead time. Simulation. Blood donation. Flexsim. 


\section{Resumen}

Objetivo: Determinar el tiempo de espera del proceso de donación de sangre en un centro de sangre y evaluar cómo varía con el aumento del número de donantes candidatos.

Metodología: Se mapearon los procesos de donación de sangre mediante técnicas de mapeo de procesos. Estos procesos se convirtieron luego en modelos de simulación de eventos discretos. Las funciones que describen cada proceso se calcularon utilizando el software Expertfit. Para identificar la correlación entre el tiempo de entrega y el número de candidatos a donación se utilizó el método del coeficiente de correlación lineal.

Originalidad / relevancia: El estudio avanza en la literatura sobre gestión de operaciones que demuestra la efectividad del método de modelado y simulación de procesos para evaluar y predecir impactos en los procesos en el contexto de la donación de sangre.

Resultados principales: Se pudo determinar el tiempo medio de espera del proceso de donación de sangre, con un nivel de confianza del 95\% y un error del 5\%. En cuanto a la correlación lineal entre las variables, el coeficiente calculado resultó en 0.3 , lo que indica que no existe correlación.

Contribuciones teóricas / metodológicas: La investigación puede demostrar mejor el uso de técnicas de modelado y simulación de sistemas para la gestión de procesos altamente estocásticos.

Palabras clave: Centro de sangre. Tiempo de espera. Simulación. Donación de sangre. Flexsim.

\section{INTRODUÇÃO}

A disponibilidade de sangue é um ponto importante para todos os sistemas de saúde, visto sua necessidade em vários tratamentos e intervenções cirúrgicas. Por exemplo, em 2012, a necessidade anual de sangue nos Estados Unidos era de cerca de 10 milhões de bolsas de sangue de $450 \mathrm{~mL}, 2,1$ na Itália e 2 na Turquia (Baş, Carello, Lanzarone e Yalçindağ, 2018). No Brasil, de acordo com o Ministério da Saúde, apenas 1,8\% da população doa sangue, porcentagem inferior à taxa recomendada pela Organização Mundial da Saúde (OMS), que é de $3 \%$ da população (Brasil, 2017).

Assim como qualquer empresa, tais instituições necessitam de boas práticas de gestão para atenderem seus clientes e manterem seus padrões de qualidades. A satisfação dos pacientes atendidos e o cuidado com a qualidade são importantes indicadores para o sucesso de qualquer empresa de saúde. Para sustentar de forma equilibrada crescimento e competência, é 
necessário que essas empresas foquem sua atenção na qualidade e eficiência dos seus serviços de maneira contínua (Buhaug, 2002).

Além disso, o uso de sangue e hemocomponentes é de responsabilidade do governo e seu custo é alto, pois necessita de tecnologia de ponta e recursos humanos altamente especializados, e tem seu fornecimento diretamente relacionado à doação voluntária (Brasil, 2010). Também, o sangue é um recurso limitado, uma vez que não pode ser produzido em laboratório, e de validade limitada, impossibilitando seu armazenamento por longo tempo (Baş et al., 2018). Essas particularidades evidenciam a necessidade de uma boa gestão dos processos da empresa dado seu alto custo de operação e da importância e fragilidade do produto que lidam.

Tais processos necessitam ser avaliados e controlados quanto à sua efetividade, eficácia, eficiência, produção, produtividade, qualidade e quanto a prevenção e redução da índices de mortalidade, além da imagem que apresenta a usuários ou clientes (Bittar, 1999). Assim, considerando que, segundo Martins e Laugeni (2005), a "produtividade" de um sistema é dada como "o quociente obtido pela divisão do quantitativo produzido por um dos fatores de produção", no caso de um hemocentro, sua produtividade pode ser entendida como o quociente resultante da divisão da quantidade de bolsas de sangue obtidas por determinado recurso utilizado.

Uma métrica útil para se analisar a produtividade de um sistema em relação ao uso do recurso "tempo" é o chamado lead time, ou "tempo de atravessamento", medida do tempo gasto pelo sistema produtivo para transformar matérias-primas em produtos acabados (Tubino, 2007), ou, na perspectiva de um doador de sangue, seria o tempo gasto pelo mesmo para realizar os procedimentos necessários para a doação desde sua chegada até sua saída do hemocentro.

Existem diversas formas de se calcular o lead time de um processo tanto em empresas de manufatura quanto de serviços, como o descrito por Tubino (2007), sendo possível encontrar diversos trabalhos recentes na literatura científica sobre a gestão desse indicador em sistemas de saúde, porém não em hemocentros (Barrios e Jiménez, 2016; Nguyen, Sivakumar e Graves, 2014; Ortiz, McClean, Nugent e Castillo, 2016). Além disso, tais métodos de cálculos podem ser integrados com técnicas de modelagem e simulação dinâmica de forma a obter diversas vantagens, sendo a de maior destaque a possibilidade de prever o efeito que determinada alteração no sistema teria sobre o mesmo e seu desempenho sem a necessidade de realizar modificações físicas dispendiosas (Freitas, 2001). 
Diante do exposto, o presente trabalho apresenta um estudo de caso do processo de doação de sangue do Hemocentro Regional de Maringá (HRM), no qual buscou-se mapear seus procedimentos, determinar o lead time dos doadores de sangue utilizando ferramentas da simulação computacional e verificar se o mesmo é influenciado pelo número de candidatos a doação de sangue em um dia.

\section{REFERENCIAL TEÓRICO}

\subsection{Cadeia de suprimento do sangue}

A cadeia de suprimento de sangue consiste nas atividades típicas de uma cadeia de suprimento com foco na produção e distribuição do sangue, sendo segmentada em quatro etapas principais: coleta, processamento, armazenamento e distribuição, com alguns autores incluindo algumas segmentações mais detalhadas em seus modelos (Baş et al., 2018; Osorio, Brailsford, Smith, Forero-Matiz e Camacho-Rodriguez, 2017; Katsaliaski e Brailsford, 2007).

Nos últimos anos, a literatura científica acerca da cadeia de suprimento do sangue tem crescido, com algumas etapas recebendo maior atenção que outras: a maior parte das pesquisas concentram-se na etapa de armazenamento, e a etapa de processamento é a menos estudada. Quanto ao estágio de coleta, diversos aspectos são abordados pelos estudos, como a configuração de pontos de coleta, políticas e métodos de coleta e situações especiais como desastres e emergências (Osorio et al., 2017). Beliën e Forcé (2011) identificaram a frequência de trabalhos publicados sobre o tema nas bases de dados científicas, conforme ilustrado na Figura 1.

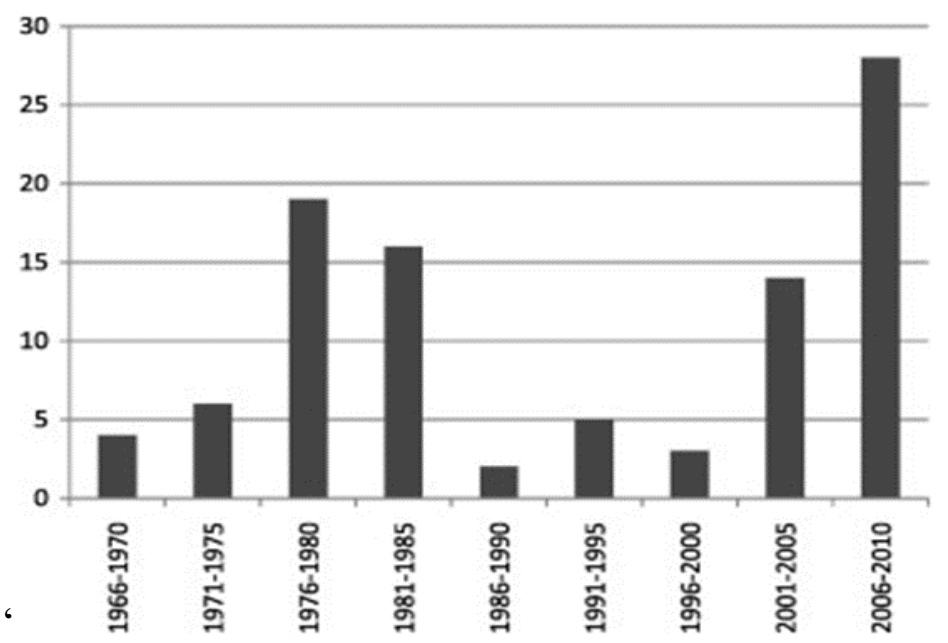

Figura 1 - Número de publicações sobre cadeia de suprimento do sangue Fonte: Beliën e Forcé (2011) 
Na etapa de coleta, um dos serviços de hemoterapia que possuem grande relevância são os hemocentros regionais, responsáveis pela coleta de sangue e componentes e por suporte às demandas de sangue estaduais (Silva, Costa e Baccara, 2015). O processo de doação de sangue em um serviço de hemoterapia como um hemocentro seguem as normas estabelecidas pela resolução RDC 153/2004, publicada em 14 de junho de 2004 pela Agência Nacional de Vigilância Sanitária (ANVISA), e revisada e atualizada pela Portaria No 2712 de 12 de novembro de 2013, e seus procedimentos são ilustrados na Figura 2.

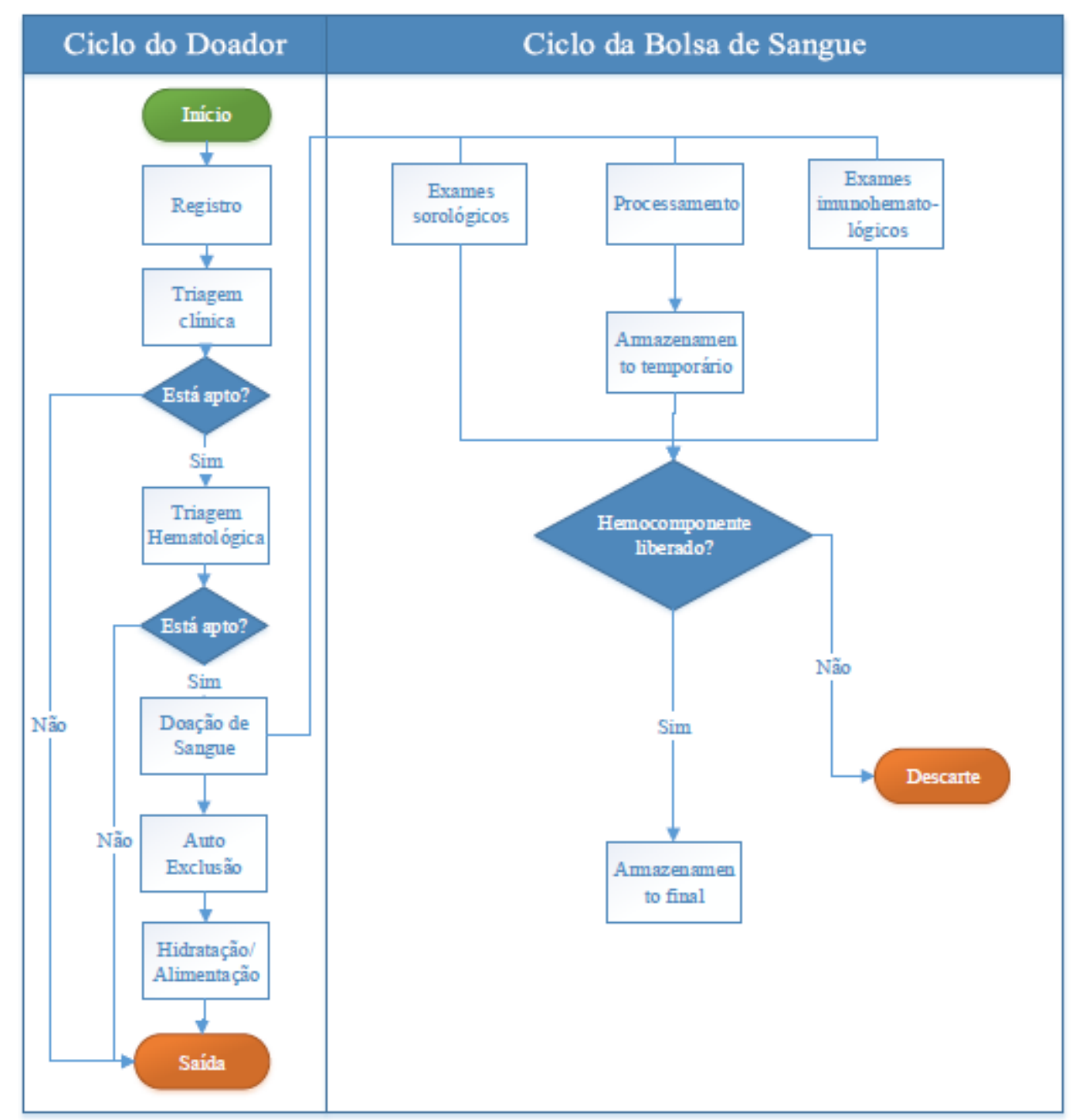

Figura 2 - Ciclo de doação do sangue

Fonte: ANVISA (2013 apud Gerônimo e Antonelli, 2017).

Um importante parâmetro para se avaliar a eficiência dos ciclos descritos na Figura 2 é o tempo necessário para a ocorrência dos mesmos (ou lead time), podendo ser calculado pelos tempos dos subprocessos individualmente, os quais são identificados por meio de técnicas de mapeamento de processos. 


\subsection{Mapeamento de processos}

O mapeamento de processos é uma ferramenta gerencial analítica e de comunicação cujo objetivo é melhorar os processos existentes de uma empresa ou organização (Hunt, 1996). Consiste na construção de um modelo que evidencia a relação entre as atividades, pessoas, dados e objetos envolvidos na produção de um produto específico.

Segundo a Association of Business Process Management Professionals (ABPM), a modelagem de processos é o conjunto de atividades envolvidas na criação de representações de processos existentes ou propostos com o propósito de refletir de maneira completa e precisa seu funcionamento. Já o modelo de um processo consiste na representação simplificada de um conceito ou atividades, podendo ser matemáticos, gráficos, físicos, narrativos ou alguma combinação destes (ABPM, 2013). Para um mesmo modelo, diversas notações podem ser utilizadas, conforme ilustrado na Figura 2a.

\begin{tabular}{|l|l|}
\hline Notação & Descrição \\
\hline $\begin{array}{l}\text { BPMN (Business Process } \\
\text { Model and Notation) }\end{array}$ & $\begin{array}{l}\text { Padrão criado pelo Object Management Group, útil } \\
\text { para apresentar um modelo para públicos-alvo } \\
\text { diferentes }\end{array}$ \\
\hline Fluxograma & $\begin{array}{l}\text { Originalmente aprovado como um padrão ANSI } \\
\text { (American National Standards Institute), inclui um } \\
\text { conjunto simples e limitado de símbolos não } \\
\text { padronizados; facilita entendimento rápido do fluxo } \\
\text { de um processo }\end{array}$ \\
\hline EPC (Event-driven Process & $\begin{array}{l}\text { Desenvolvido como parte da estrutura de trabalho } \\
\text { ARIS, considera eventos como "gatilhos para" ou } \\
\text { "resultados de" uma etapa do processo; útil para }\end{array}$ \\
\hline modelar conjuntos complexos de processos
\end{tabular}

Figura 2a - Notações de modelagem de processos

Fonte: $\operatorname{ABPMN}(2013$, p. 79).

Segundo Kalman (2002), para se realizar um mapeamento de um processo são necessárias oito etapas: 
1. Pré-mapeamento: Gestores identificam os problemas ou questões críticas do negócio, selecionam um proprietário do processo e definem uma meta mensurável;

2. Construir um mapa de processos macro;

3. Identificar gargalos e problemas no processo existente;

4. Identificar quais dos gargalos e problemas no processo existente são prioritários;

5. Construir um mapa com os subprocessos selecionados e identificar a causa raiz dos problemas;

6. Reconstruir o mapa;

7. Desenvolver planos de ação para aprovação dos gestores;

8. Implementar os planos aprovados.

$\mathrm{Na}$ área da saúde, diversos trabalhos exemplificam a utilidade do mapeamento de processos como auxílio para gestão. Vaccari, Montasser, Tudor e Leone (2017) mapeiam os processos de um hospital para identificar sua geração de resíduos e melhor geri-los. Chung, Hunter-Smith, Spychal, Ramakrishnan e Rozen (2017) traz uma revisão de diversos trabalhos científicos sobre o uso de mapeamentos de processos em cirurgias para aumentar a eficiência destas. Takagi, Kanai e Misue (2017) mapeiam o fluxo dos pacientes obstétricos para a construção de um modelo matemático que melhor o represente. Gurupur, Suh, Selvaggi, Karla, Nair, e Ajit, (2012) utilizam técnicas de mapeamento para a construção e um sistema de informação em uma empresa de saúde.

Construído o mapa dos processos e mensurados os tempos necessários das operações, as informações podem ser agregadas e estudadas utilizando simulação computacional, bem como a observação de melhorias propostas tanto na ordem dos processos quanto nos tempos destes.

\subsection{Simulação computacional}

Diversos conceitos de "simulação" podem ser encontrados na literatura, sendo um dos mais difundidos o de Bateman, Bowden, Gogg, Harrel, Mott, e Montevechi (2013):

Simulação é um processo de experimentação com um modelo detalhado de um sistema real para determinar como o sistema responderá a mudanças em sua estrutura, ambiente ou condições de contorno. 
Assim, a simulação pode ser entendida como a replicação de sistemas por meio de modelos de forma a possibilitar a avaliação de seu estado atual e futuro após alguma alteração no sistema estudado. Modelos, à vista da computação, são "programadas de computadores cujas variáveis apresentam o mesmo comportamento dinâmico e estocástico do sistema real que apresenta" (Shannon, 1975).

No tocante aos hemocentros, diversos trabalhos utilizam a simulação computacional como método para resolução de problemas e otimização de processos. Lowalekar e Ravichandran (2010) utilizaram modelos de simulação para comparar o efeito que a introdução de dois critérios de seleção de doadores teria sobre os indicadores de custo, desperdícios e disponibilidade de bolsas em relação à política de coletar toda a oferta disponível de bolsas de sangue. Alfonso, Xie, Augusto e Garraud (2012, 2013) desenvolveram modelos de simulação objetivando determinar a capacidade de um serviço de coleta de sangue e os recursos humanos demandados. Testik, Ozkaya, Aksu e Ozcebe (2012) utilizaram técnicas da modelagem e simulação para, a partir dos dados de chegada de doadores em um hemocentro, calcular os recursos humanos necessários e sua alocação no processo de doação de sangue. Alfonso, Xie, e Augusto, (2015) apresentaram uma abordagem de otimização baseada na simulação da dinâmica de eventos para planejamento de capacidade e agendamento de consultas em serviços de hemoterapia.

Para a construção de uma simulação, Bateman et al. (2013) e Freitas (2001) propõem uma sequência de passos que se inter-relacionam conforme Figura 3, tomando como base a sequência de Banks e Carson (1984). 


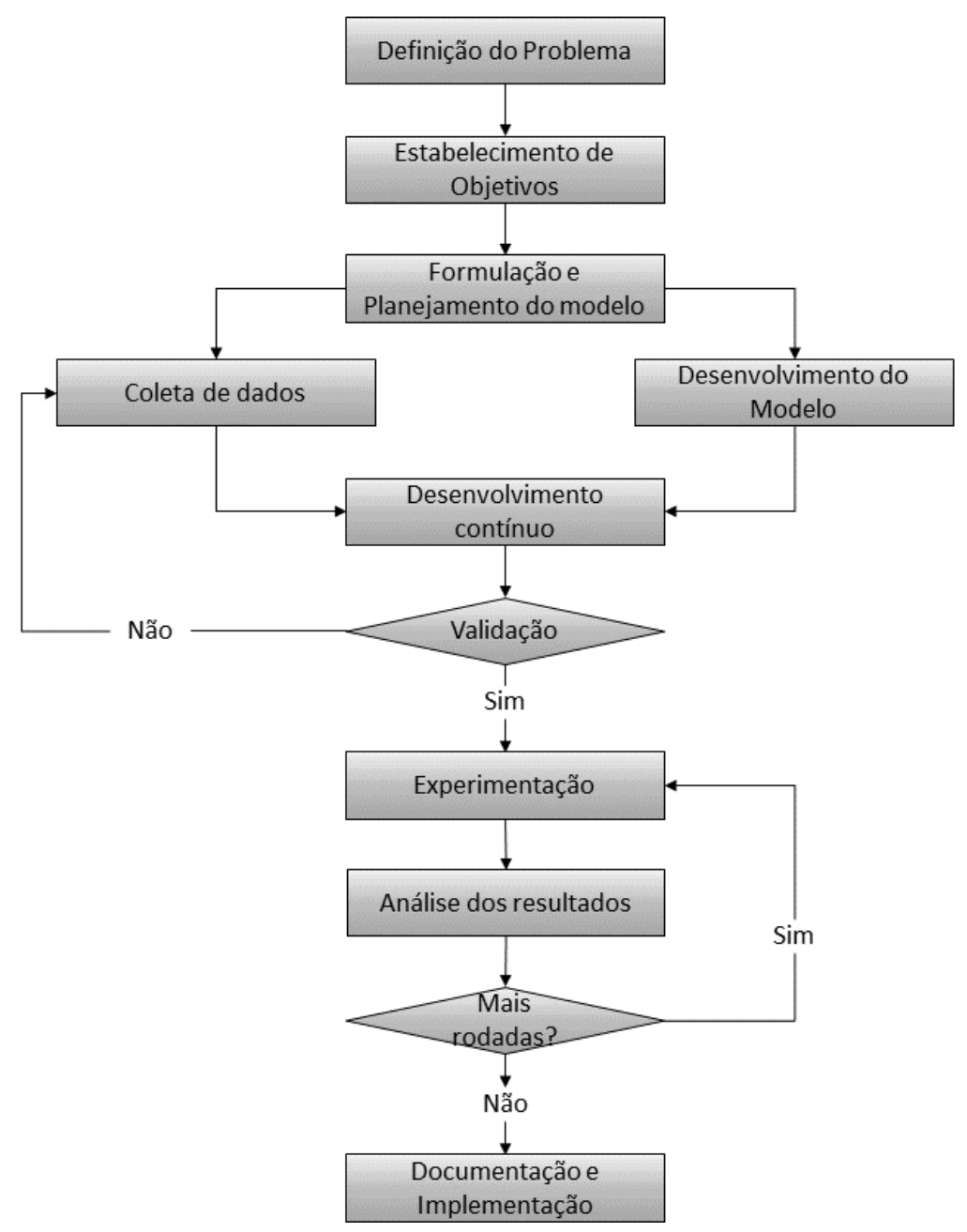

Figura 3 - Interrelação entre os passos da simulação Fonte: Adaptado de Bateman et al. (2013, p.33).

1. Definição do Problema e Estabelecimento de Objetivos: Mesmo os mais exaustivos estudos serão desvalorizados se não visarem a necessidade real (Bateman et al., 2013). Nesse passo, deve-se entender o porquê de o problema estar sendo estudado, quais respostas esperam-se alcançar e quais restrições e limites são esperados das soluções obtidas (Freitas, 2001);

2. Formulação e Planejamento do Modelo: Traçar um esboço do sistema, de forma gráfica ou algorítmica, definindo componentes e descrevendo as variáveis e interações lógicas que constituem o sistema (Freitas, 2001);

3. Coleta de Dados: Coletar fatos, informações e estatísticas derivados de observações, experiências pessoais ou de arquivos históricos (Freitas, 2001). Se dados insuficientes ou incompletos estão disponíveis, também é necessário que o responsável pelo projeto de simulação: consiga assistência adicional daqueles 
familiarizados com o sistema; reúna informações por si próprio; e apoiar-se em pressupostos na construção do modelo, sendo necessário uma análise de sensibilidade posteriormente nesse último caso (Bateman et al., 2013);

4. Desenvolvimento do Modelo: Codificar o modelo numa linguagem de simulação apropriada (Freitas, 2001). O modelo normalmente inicia-se como uma abstração conceitual do sistema, com crescentes níveis de detalhes adicionados durante seu desenvolvimento, tornando-se mais lógico à medida que o processamento de eventos e os relacionamentos entre estes estejam bem definidos (Bateman et al., 2013);

5. Validação: Assegurar que o modelo reflete a operação do sistema real em estudo de tal forma que dê encaminhamento ao problema definido, funcionando da maneira como o modelador pretendia (Bateman et al., 2013). Os resultados fornecidos pelo modelo devem possuir crédito e ser representativos dos resultados do modelo real (Freitas, 2001);

6. Experimentação: Projetar um Projeto Experimental (conjunto de experimentos que produza a informação desejada de forma a possibilitar a análise e comparação de alternativas) e executar as simulações para a geração dos dados desejados (Freitas, 2001; Bateman et al., 2013);

7. Análise dos Resultados: Traçar inferências sobre os resultados alcançados pela simulação e comparações para as medidas de desempenho nos cenários planejados (Freitas, 2001). Em certos casos, podem ser necessárias replicações adicionais para se determinar a significação estatística de uma melhoria observada (Freitas, 2001; Bateman et al., 2013);

8. Implementação: Programação do projeto de implantação das decisões tomadas. Nessa etapa, a boa comunicação entre a equipe do projeto é fundamental para o bom desenvolvimento deste (Freitas, 2001; Bateman et al., 2013).

Diversos softwares podem ser utilizados para a construção da simulação. Um deles é o Flexsim ${ }^{\circledR}$, seu módulo adicional Flexsim Healthcare ${ }^{\circledR}$ e a possibilidade de integrá-lo com o software ExpertFit@, descritos a seguir. 


\subsubsection{Softwares utilizados}

O software de simulação Flexsim ${ }^{\circledR}$ foi lançado em 2003 e possui um ambiente de programação orientada a objeto. Nele, há um módulo adicional específico para simulação de sistemas de saúde chamado Flexsim Healthcare®, ou apenas Flexsim HC, o qual permite seu usuário analisar todos os componentes do atendimento ao paciente e avaliar o impacto que eles têm no sistema de saúde (Flexsim, 2018).

O Flexsim HC possui integração com o ExpertFit®, software de ajuste de distribuição que possibilita encontrar distribuições estatísticas de maneira ágil, determinando automática e precisamente qual a distribuição de probabilidade melhor representa a base de dados estudada em poucos segundos. Tais distribuições possuem diferentes representações para serem inseridas no Flexsim HC de acordo com seus parâmetros de entrada, conforme exemplificado na Figura 4, na qual algumas distribuições são descritas.

\begin{tabular}{|c|c|c|}
\hline Distribuição & Representação no Flexsim HC & Parâmetros de entrada \\
\hline Beta & $\operatorname{beta}\left(a, b, \alpha_{1}, \alpha_{2}\right.$, stream $)$ & $\begin{array}{ll}- & \mathrm{a}=\text { extremo inferior } \in(-\infty, \infty) \\
- & \mathrm{b}=\text { extremo superior }(\mathrm{b}>\mathrm{a})\end{array}$ \\
\hline Johnson $\mathrm{S}_{\mathrm{B}}$ limitada & johnsonbounded(a, b, $\alpha_{1}, \alpha_{2}$, stream $)$ & $\begin{array}{l}\text { - } \alpha_{1}=1^{\circ} \text { parâmetro de forma } \in(0, \infty) ; \\
\text { - } \quad \alpha_{2}=2^{\circ} \text { parâmetro de forma } \in(0, \infty) ; \\
\text { stream }=\text { semente de geração de } \\
\text { números aleatórios } \in\{0,1,2, \ldots\} .\end{array}$ \\
\hline Erlang & $\operatorname{erlang}(\gamma, \beta, \mathrm{m}$, stream $)$ & \multirow{3}{*}{$\begin{array}{ll}- & \gamma=\text { parâmetro de localização } \in(-\infty, \\
\infty) ; & \beta=\text { parâmetro de escala }(\beta>0) ; \\
- & \mathrm{m}=\text { parâmetro de forma } \in\{1,2, \ldots\} ; \\
- & \text { stream }=\text { semente de geração de } \\
\text { números aleatórios } \in\{0,1,2, \ldots\} .\end{array}$} \\
\hline Log-Normal & $\operatorname{lognormal} 2(\gamma, \beta, \mathrm{m}$, stream $)$ & \\
\hline Pearson tipo V & pearsont $5(\gamma, \beta, \mathrm{m}$, stream $)$ & \\
\hline Exponencial & $\operatorname{exponential}(\gamma, \beta$, stream $)$ & $\begin{array}{ll}- & \gamma=\text { parâmetro de localização } \in(-\infty, \\
\infty) ; & \\
- & \beta=\text { parâmetro de escala }(\beta>0) ; \\
- & \text { stream }=\text { semente de geração de } \\
\text { números aleatórios } \in\{0,1,2, \ldots\} .\end{array}$ \\
\hline Pearson tipo VI & pearsont $6\left(\gamma, \beta, \alpha_{1}, \alpha_{2}\right.$, stream $)$ & $\begin{array}{ll}- & \gamma=\text { parâmetro de localização } \in(-\infty, \\
\infty) ; & \\
- & \beta=\text { parâmetro de escala }(\beta>0) ; \\
- & \alpha_{1}=1^{\circ} \text { parâmetro de forma } \in(0, \infty) ; \\
- & \alpha_{2}=2^{\circ} \text { parâmetro de forma } \in(0, \infty) ;\end{array}$ \\
\hline
\end{tabular}




\begin{tabular}{|c|c|c|}
\hline & & $\begin{array}{l}-\quad \text { stream }=\text { semente de geração de } \\
\text { números aleatórios } \in\{0,1,2, \ldots\} .\end{array}$ \\
\hline Weibull & weibull $(\gamma, \beta, \alpha$, stream $)$ & $\begin{array}{ll}- & \gamma=\text { parâmetro de localização } \in(-\infty, \\
\infty) ; & \\
- & \beta=\text { parâmetro de escala }(\beta>0) ; \\
- & \alpha=\text { parâmetro de forma }(\alpha>0) ; \\
- & \text { stream }=\text { semente de geração de } \\
\text { números aleatórios } \in\{0,1,2, \ldots\} .\end{array}$ \\
\hline
\end{tabular}

Figura 4 - Representações de distribuições estatísticas no Flexsim HC e seus parâmetros de entrada.

Fonte: Flexsim (2018).

\subsection{Intervalo de confiança da simulação}

Segundo Freitas (2001), a determinação de intervalos de confiança para as variáveis estudadas num sistema simulado é um componente fundamental para se analisar os resultados da simulação. Tal intervalo compreende uma faixa numérica que possui uma probabilidade igual a $(1-\alpha)$ de incluir o verdadeiro valor da variável sob análise, onde $(1-\alpha)$ é denominado o nível de confiança do intervalo e $\alpha$ é o erro admitido ao se concluir sobre a presença do verdadeiro valor da variável no intervalo calculado.

Ainda segundo o autor, para se calcular tal intervalo é necessário calcular a média e o desvio padrão da variável a partir de uma amostra. Porém, para isso, é necessário primeiramente determinar o tamanho dessa amostra, o que corresponde, no caso da simulação computacional, determinar o número de replicações da simulação (n). Esse processo pode ser realizado iterativamente por meio dos passos a seguir descritos por Freitas (2001):

1. Definir o nível de confiança $(1-\alpha)$ do intervalo da variável que se deseja obter;

2. Executar a simulação com $n^{*}$ replicações, podendo o valor de $n^{*}$ para a primeira iteração ser um valor arbitrário;

3. Calcular a média $\bar{x}$ e o desvio padrão s da variável analisada após as $\mathrm{n}^{*}$ replicações;

4. Aplicar a Eq. 01 abaixo e determinar o valor de n, ou seja, do tamanho da amostra necessário para se obter um intervalo de confiança com o nível de confiança exigido, considerando um erro de r\% e o valor $\mathrm{z}$ da variável Normal padronizada para o nível de confiança desejado: 


$$
n=\left(\frac{100 z s}{r \bar{x}}\right)^{2}
$$

5. Se $\mathrm{n} \leq \mathrm{n}^{*}$, o número de replicações já realizada é suficiente. Do contrário, no caso de $n>n^{*}$, realizar uma nova iteração a partir do passo 2, substituindo o valor de $n^{*}$ pelo de $n$.

Com o tamanho da amostra definido, pode-se determinar o intervalo de confiança calculando o semi-intervalo h por meio da Eq. 02.

$$
h=t_{n-1,1-(\alpha / 2)} \frac{s}{\sqrt{n}}
$$

Onde $\mathrm{t}_{\mathrm{n}-1,1-\alpha / 2}$ é o valor tabulado de distribuição $\mathrm{T}$ de Student. Obtido o valor de $\mathrm{h}$ e sabendo que o intervalo é simétrico em torno da média, os limites do intervalo são:

$$
[\bar{X}-h, \bar{X}+h]
$$

Como pode-se observar, o tamanho do intervalo depende do nível de confiança desejado, de forma que, quanto maior esse nível de confiança, maior o tamanho do intervalo. Além de determinar valores confiáveis para as análises, para estudos em que se busca observar a influência de uma determinada variável em outra em um sistema é necessário realizar uma análise de correlação.

\subsection{Análise de correlação}

Quando se possuem duas variáveis quantitativas e deseja-se descobrir se há dependência entre elas (ou seja, se a variação no valor de uma das variáveis influencia na variação da outra variável), um procedimento bastante comum é o uso de gráficos de dispersão, que consiste em representar os pares de valores das variáveis analisadas num sistema cartesiano e observar como o aumento ou diminuição de uma das variáveis influencia na outra (Bussab e Morettin, 2017).

Ainda segundo Bussab e Morettin (2017), muitas vezes pode ser útil quantificar a associabilidade entre as variáveis, o que pode ser muito difícil de se realizar apenas por meio da observação dos gráficos de dispersão. Assim, existem diversos procedimentos para se determinar essa associabilidade, cuja mais simples é a linear. No procedimento para se determinar esse tipo de associação, busca-se calcular um coeficiente que avalia o quanto os pontos plotados no gráfico de dispersão aproximam-se de uma reta, chamado de coeficiente de correlação linear ou somente coeficiente de correlação, o qual é calculado segundo a Eq. 03: 
Na qual:

$$
\operatorname{Corr}(X, Y)=\frac{1}{n} \sum_{i=1}^{n}\left(\frac{x_{i}-\bar{x}}{D P(X)}\right)\left(\frac{y_{i}-\bar{y}}{D P(Y)}\right)
$$

- $\operatorname{Corr}(\mathbf{X}, \mathbf{Y})$ : coeficiente de correlação entre as variáveis X e Y;

- $\quad$ n: número de pares de valores $\left(\mathrm{x}_{1}, \mathrm{y}_{1}\right),\left(\mathrm{x}_{2}, \mathrm{y}_{2}\right) \ldots\left(\mathrm{x}_{\mathrm{n}}, \mathrm{y}_{\mathrm{n}}\right)$;

- DP(X), DP(Y): desvio padrão do rol de valores das variáveis $\mathrm{X}$ e Y, respectivamente.

Em seguida, avalia-se o valor do coeficiente, que varia entre -1 e 1, conforme a Figura 5 ilustra.

CORRELAÇ̃̃O NEGATIVA

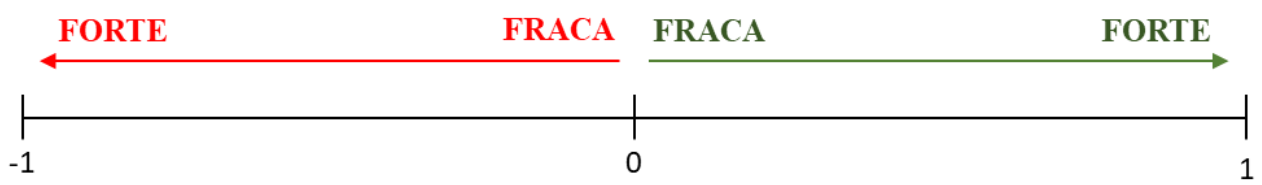

Figura 5 - Tipos e níveis de correlação entre duas variáveis de acordo com o coeficiente de correlação

Fonte: Autoria própria (2018).

Quando a correlação é positiva, o aumento de uma das variáveis causa o aumento da outra. Já quando é negativa, o aumento de uma das variáveis causa a diminuição da outra. E quanto mais próximo de zero, menor a dependência entre as variáveis (Bussab e Morettin, 2017).

\section{MÉTODO}

Considerando-se os aspectos do problema deste trabalho, o método de pesquisa mais apropriado para investigá-lo foi a modelagem, na qual se supõe que é possível construir modelos que representem total ou parcialmente o comportamento dos processos operacionais de ambientes reais estudados e que capturem os problemas de tomada de decisão envolvidos nesses processos (Bertand \& Fransoo, 2009; Morabito \& Pureza, 2018). Como amostra de estudo, foram utilizadas as pessoas e processos envolvidos e existentes para doação de sangue no HRM. Assim, para determinar o lead time desse processo, com base na literatura apresentada na Seção Erro! Fonte de referência não encontrada. e na estrutura proposta por Morabito e Pureza (2018) para condução do método de modelagem, as seguintes etapas foram executadas:

1. Definir o problema: Definir o escopo do problema em estudo, as decisões de interesse, os objetivos envolvidos, e o modelo conceitual do problema. 
1.1. Caracterizar o problema: Em reuniões com colaboradores do hemocentro e com pesquisadores do grupo, caracterizou-se o processo de doação de sangue e as suas nuances;

1.2. Modelar processos: Identificar elementos e fases envolvidos no processo de doação de sangue, bem como a relação entre cada;

1.3. Construir mapa de processos: Desenhar o processo de doação de sangue utilizando linguagem BPMN e o software Bizagi Modeler®;

1.4. Validar mapa de processos: Validar com colaboradores do HRM se o mapa do processo correspondia à realidade;

1.5. Identificar dados de entrada necessários: Com base no modelo, identificar os tempos e frequências que deveriam ser coletadas para alimentar a simulação;

2. Construir o modelo: As informações e estimativas coletadas na primeira fase foram utilizadas para desenvolver e avaliar um modelo matemático do problema, representado por meio de lógicas simulação.

2.1. Construir modelo de simulação: Codificar o mapa de processos em um modelo de simulação utilizando o software Flexsim HC;

2.2. Coletar dados por filmagens: Coletar os tempos e as frequências de ocorrência de cada evento e procedimento por meio das câmeras de segurança, conforme identificados na etapa anterior;

3. Solucionar o modelo: Alimentar a simulação com os dados de entrada e executar os experimentos necessários para atingimento do objetivo proposto.

3.1. Alimentar simulação com dados de entrada: Alimentar a simulação com as funções de distribuição estatísticas obtidas na etapa 7, bem como jornadas de trabalho e demais variáveis para refinamento da simulação;

3.2. Identificar distribuições estatísticas: Identificar quais distribuições estatísticas melhor descrevem a ocorrência de cada evento e a variação dos tempos de cada procedimento utilizando o software ExpertFit $®$;

3.3. Realizar experimentos: Com base no número de experimentos necessários para se identificar o lead time do processo, definido pelo tamanho ideal da amostra segundo Freitas (2001), a simulação foi executada por tal número e seus resultados foram coletados. 
4. Validação da simulação: Assegurar que o modelo refletisse a operação do sistema real em estudo, comparando as variáveis de saída simuladas com as quantidades reais;

5. Implementação da solução: Converter os resultados matemáticos em decisões operacionais.

5.1. Determinar o lead time de uma amostra de dias: Somar os tempos necessários para a realização de cada procedimento do processo de doação de sangue e determinar o lead time e seu intervalo de confiança, com um certo nível de confiança estatística;

5.2. Análise de correlação: calcular o coeficiente de correlação entre as variáveis lead time e o número de candidatos a doação de sangue.

\section{RESULTADOS E DISCUSSÕES}

\subsection{Modelagem de processos}

Em entrevistas com os colaboradores do HRM, foram identificados três processos: a doação de sangue, a doação por aférese e a coleta de sangue para doação de medula, os quais foram mapeados e são detalhados nos tópicos 4.1.1, 4.1.2 e 4.1.3 seguintes. Embora este trabalho busque estudar o processo de doação de sangue apenas, os outros dois processos citados interferem no seu lead time, sendo assim necessário estudá-los também.

\subsubsection{Doação de sangue}

É a doação de sangue habitual, também chamada de doação de sangue "total", onde até $450 \mathrm{ml}$ de sangue são coletados em uma bolsa produzida com materiais e soluções que permitem a preservação do sangue (Hemonorte, 2013), sendo o procedimento mais frequente do HRM, representado na Figura 6 e cujo lead time foi determinado. 


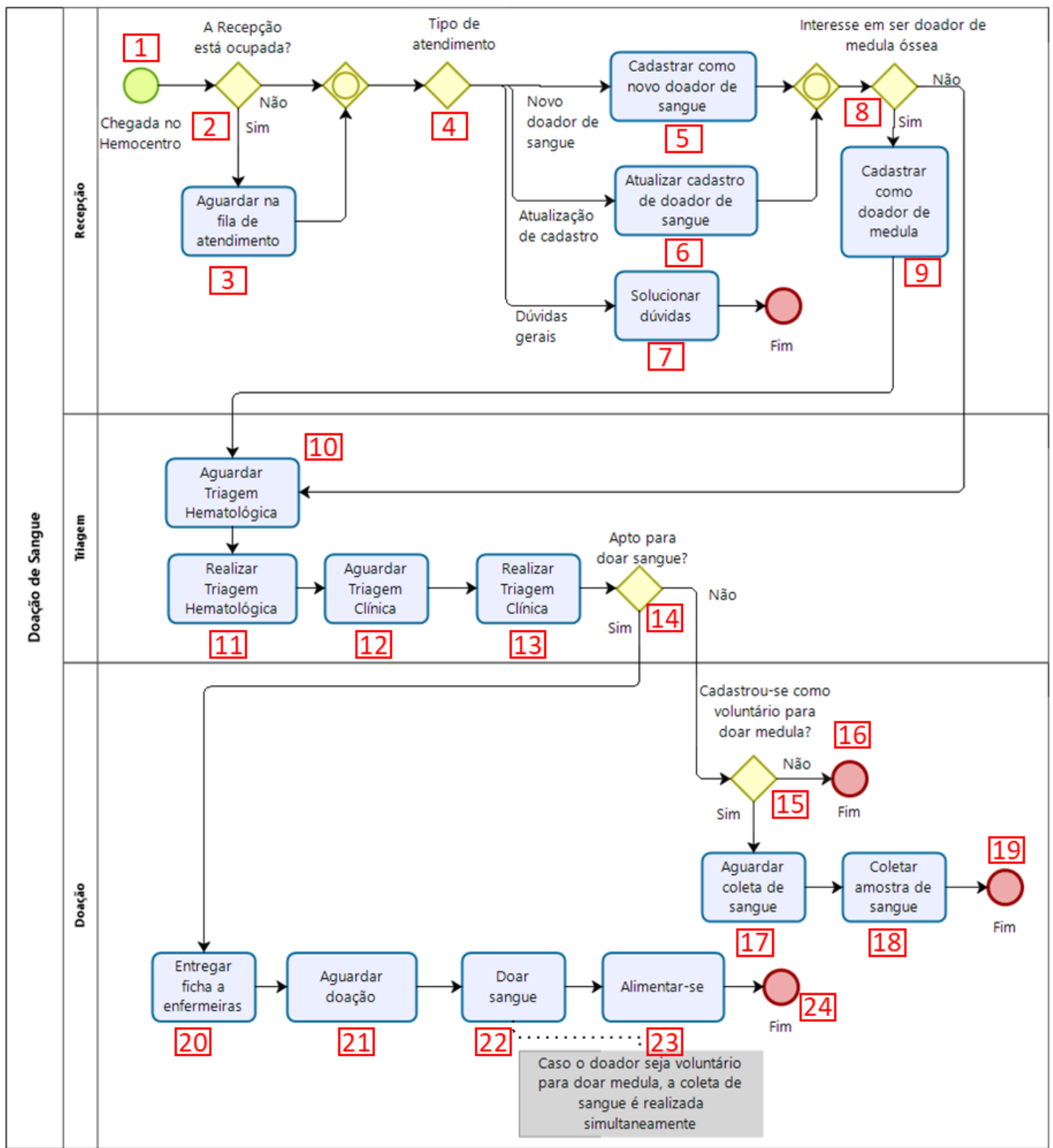

Figura 6 - Processo de um doador de sangue Fonte: Autoria própria (2018).

O fluxograma da Figura corresponde tanto aos doadores que objetivam apenas doar sangue como para os que objetivam doar sangue e medula. Nesse último caso, uma vez que o interessado em doar medula necessita que parte de seu sangue seja coletado para análises de compatibilidade, tal coleta pode ocorrer simultaneamente à doação de sangue.

\subsubsection{Doação por aférese}

Na doação de sangue por aférese, o sangue é retirado do braço do doador por meio de um kit estéril e descartável, instalado em um equipamento separador de células. O 
componente desejado é então drenado para uma bolsa de coleta e os demais componentes vão retornando continuamente ao doador (Paraná, 2013).

No HRM, o processo de um doador por aférese é semelhante ao do doador de sangue habitual, com a exceção de que, na doação por aférese, o doador sempre já terá cadastro no HRM e de que a etapa de coleta de hemocomponentes é substancialmente mais longa que a etapa de doação de sangue. O fluxograma do processo desse doador encontra-se na Figura 7. Os números em vermelho identificam cada subprocesso e decisão e suas funções serão detalhadas na Seção 4.2.

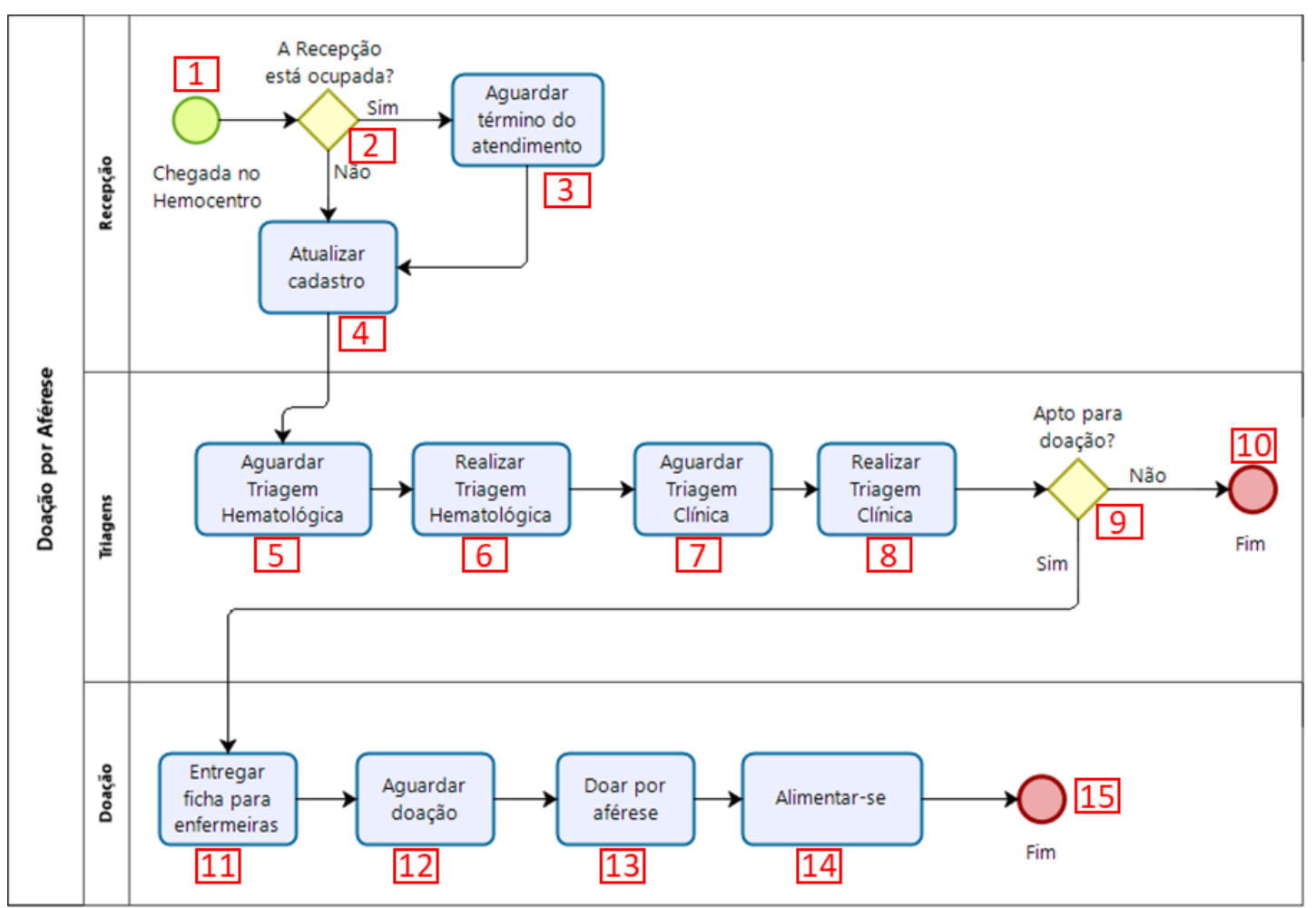

Figura 7 - Processo de um doador por aférese Fonte: Autoria própria (2018).

Convém ressaltar que o doador por aférese possui prioridade em relação aos outros doadores na fila de atendimento. Logo, caso um doador por aférese chegue ao HRM e haja fila para atendimento na Recepção, ele ocupa o primeiro lugar da fila, sendo o próximo doador a ser atendido, caso a Recepção esteja ocupada no momento da chegada, ou imediatamente, caso a Recepção esteja vazia. 


\subsubsection{Coleta de sangue para doação de medula óssea}

O HRM também realiza coletas de sangue para doação de medula óssea, havendo a possibilidade de uma pessoa interessar-se em doar apenas medula óssea, não realizando a doação de sangue simultaneamente. Conforme exposto no site do Instituto Nacional de Câncer José Alencar Gomes da Silva (INCA), nesse processo, os interessados em serem doadores de medula tem uma pequena amostra de sangue coletada $(10 \mathrm{ml})$ para fins de tipagem de sangue e cadastro e, caso seja encontrado um doador compatível, uma nova coleta é realizada para fins de confirmação de compatibilidade (INCA, 2015). Essa primeira coleta de sangue também pode ocorrer durante a doação de sangue, caso o voluntário também seja doador de sangue e não seja reprovado durante as triagens.

No HRM, em ambas as coletas, o processo segue o fluxograma descrito na Figura 8 e o doador possui prioridade na fila de atendimento, semelhantemente à doação por aférese.

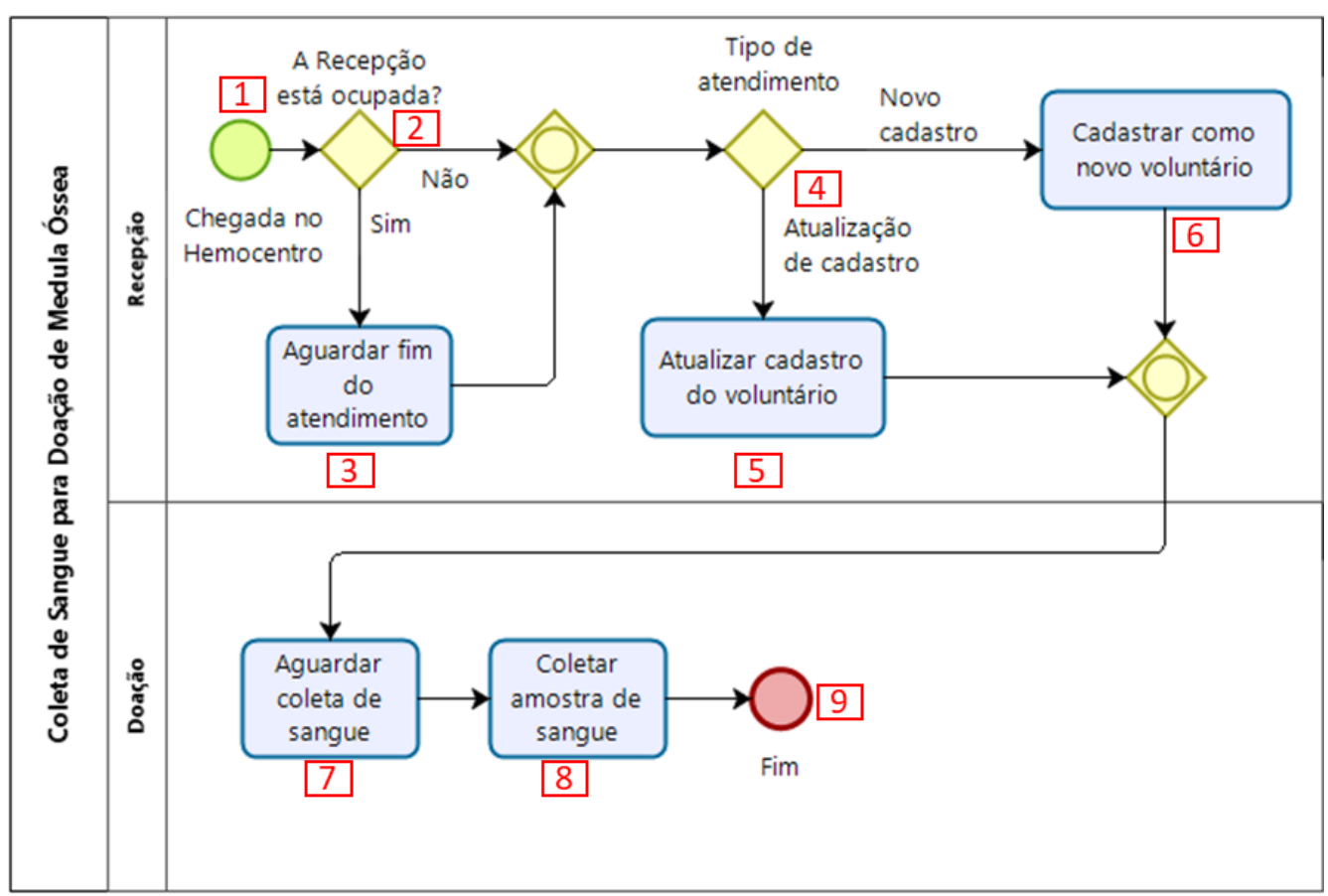

Figura 8 - Processo de coleta de sangue de um doador de medula óssea. Fonte: Autoria própria (2018).

\subsection{Apresentação e validação do modelo}

Para representar os processos apresentados nos tópicos anteriores, construiu-se o modelo da simulação no software Flexsim HC, ilustrado na Figura 9. 


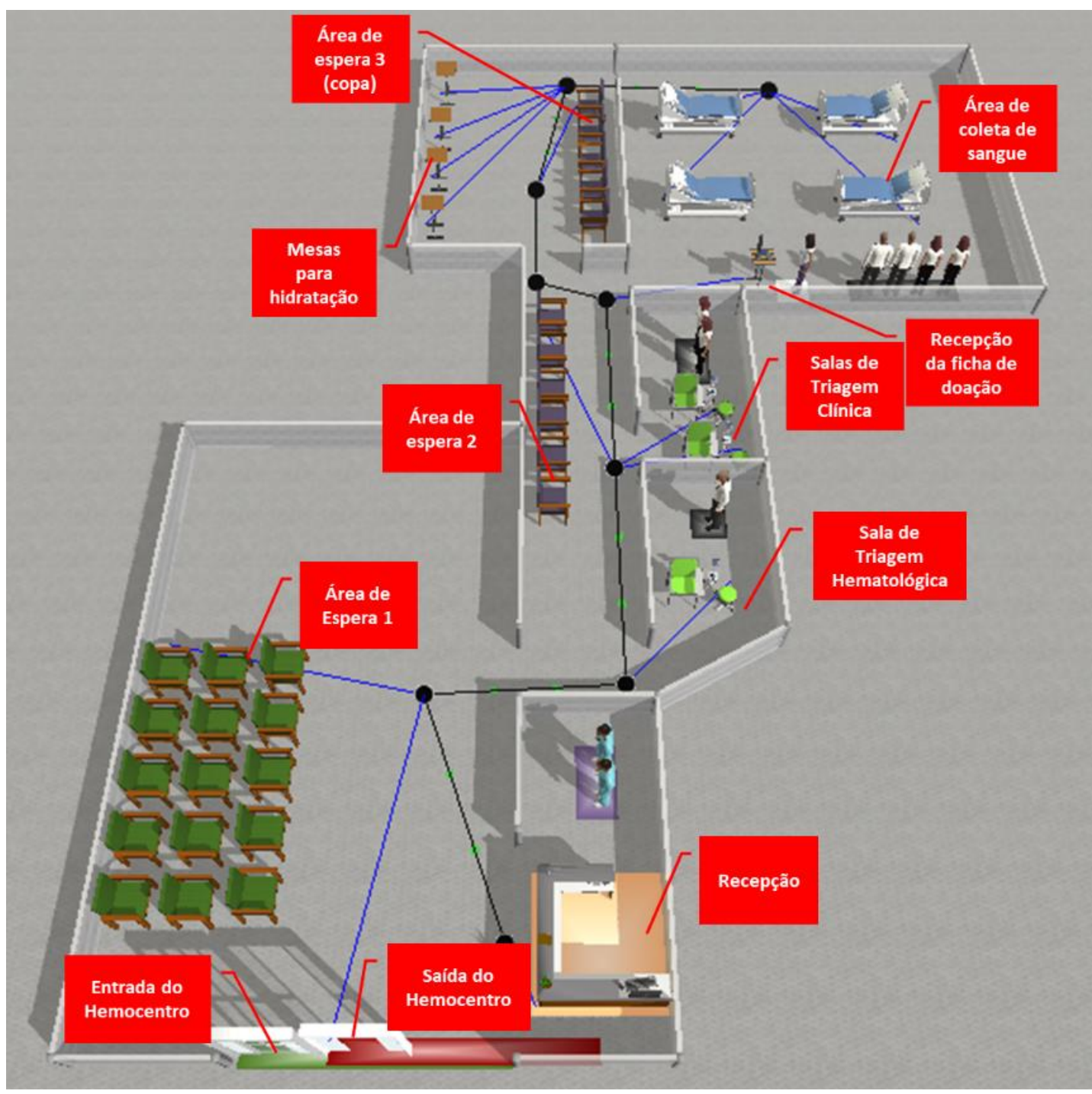

Figura 9 - Modelo do HRM construído no Flexsim HC.

Fonte: Autoria própria (2018).

Os quadros em vermelho ilustrados na Figura indicam os elementos do modelo em que ocorrem cada subprocesso e decisão enumerados na Figura, Figura e Figura . A relação entre quais subprocessos e decisões pertinentes a cada tipo de doador ocorrem em cada elemento do modelo está disposta na Figura 10. 


\begin{tabular}{|c|c|c|c|}
\hline \multirow[t]{2}{*}{ Descrição } & \multicolumn{3}{|c|}{ Subprocessos e decisões } \\
\hline & Doação de sangue & Doação por aférese & $\begin{array}{c}\text { Coleta de sangue } \\
\text { para doação de } \\
\text { medula }\end{array}$ \\
\hline Entrada do Hemocentro & 1 & 1 & 1 \\
\hline Área de espera 1 & 3 e 10 & 3 e 5 & 7 \\
\hline Recepção & $2,4,5,6,7,8,9$ e 15 & 2 e 4 & $2,3,4,5$ e 6 \\
\hline Sala de triagem hematológica & 11 & 6 & - \\
\hline Área de espera 2 (Corredor) & 12 e 17 & 7 & - \\
\hline Salas de triagem clínica & 13 e 14 & 8 e 9 & - \\
\hline Recepção da ficha de doação & 20 & 11 & - \\
\hline Área de espera 3 (Copa) & 21 & 12 & - \\
\hline Área de coleta de sangue & 18 e 22 & 13 & 8 \\
\hline Mesas para hidratação & 23 & 14 & - \\
\hline Saída do Hemocentro & 16 e 19 & 10 e 15 & 9 \\
\hline
\end{tabular}

Figura 10 - Relação entre subprocessos e decisões de cada tipo de doador com onde ocorrem no modelo de simulação.

Fonte: Autoria própria (2018).

Para configurar a chegada de cada doador considerando as especificidades de cada, foram utilizadas duas funções: a função "Shift Schedules", que permite definir qual o horário de funcionamento do HRM, e as próprias configurações do elemento "Entrada do Hemocentro", cujos campos são descritos na Figura 11

Figura a seguir. 


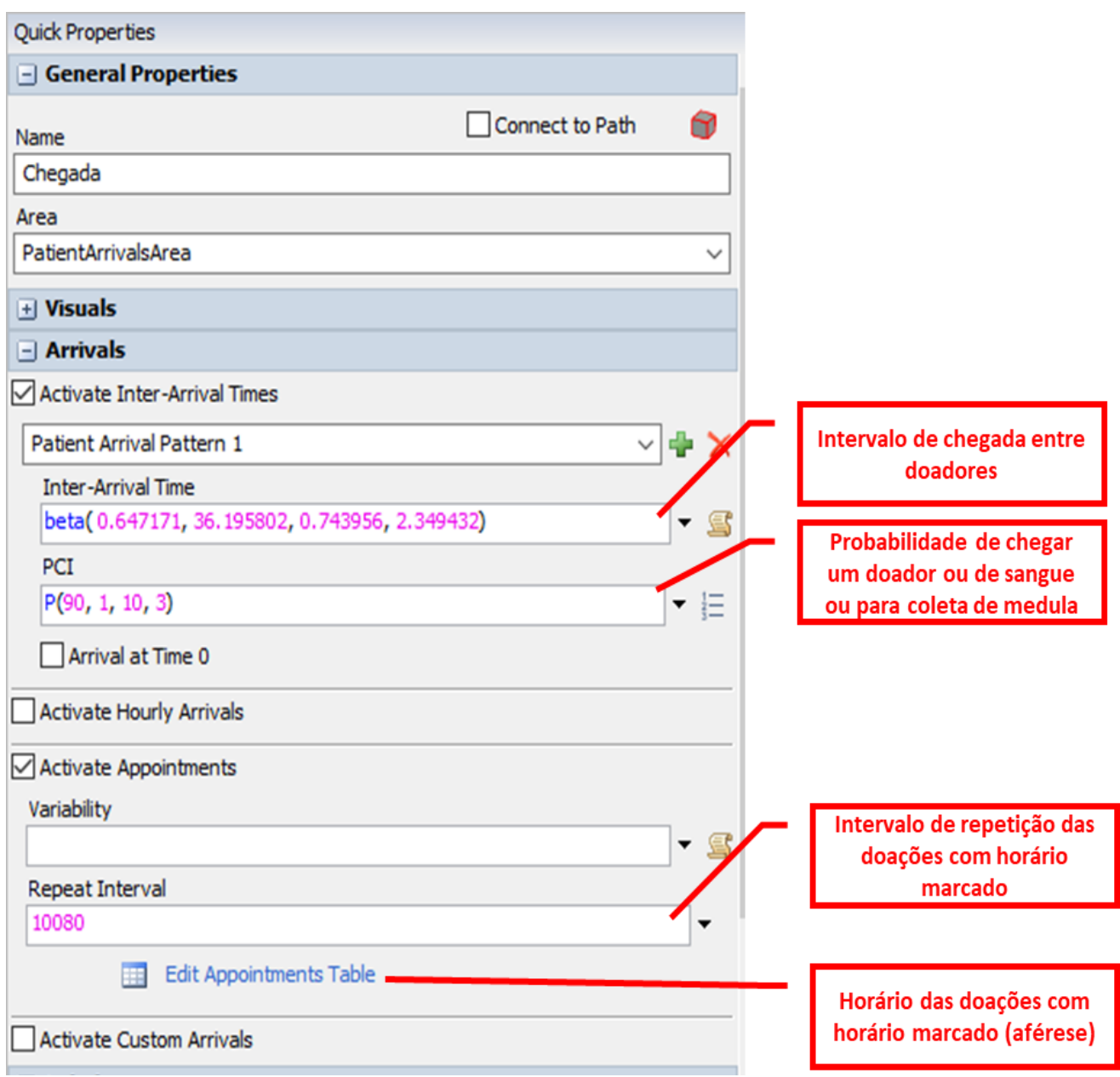

Figura 11 - Campos do elemento "Entrada do Hemocentro" utilizados no modelo. Fonte: Autoria própria (2018).

Para inserir as configurações de cada processo, foram utilizados os campos descritos na Figura 12, que ilustra um elemento de processamento. 


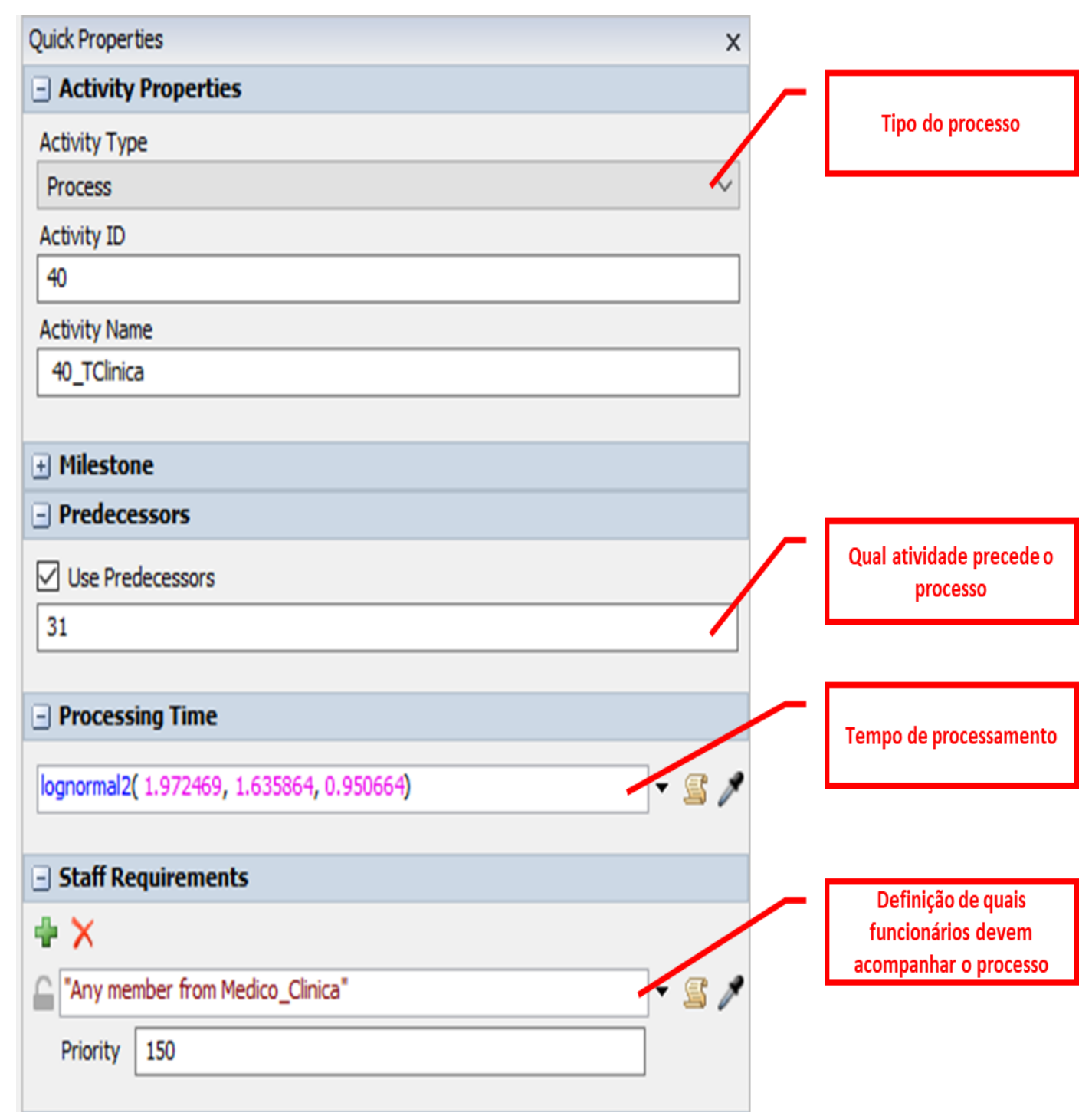

Figura 12 - Campos de um elemento de processamento utilizados no modelo. Fonte: Autoria própria (2018).

Por fim, para as decisões do processo, foram utilizados elementos de processo do tipo “Decision Point”, cujos campos são ilustrados na Figura 13. 


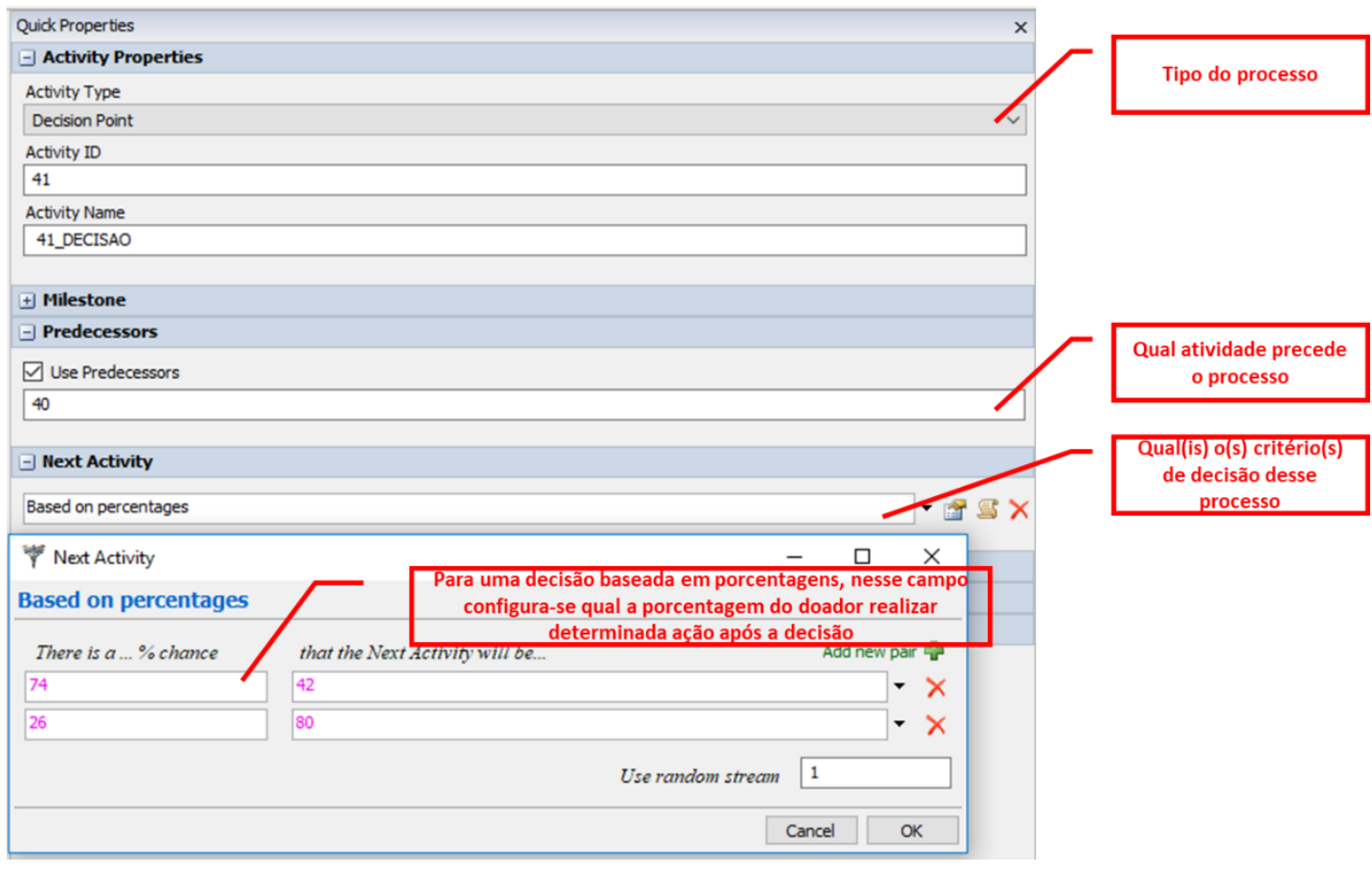

Figura 13 - Campos do elemento de processo do tipo "Decision Point" utilizados no modelo. Fonte: Autoria própria (2018).

O HRM forneceu as filmagens de 6 dias de doação. Para os tempos de cada processo, foram coletados os tempos durante 1 dia de trabalho em seu horário de operação normal (das 07:00 às 18:30).

Tais tempos foram inseridos no software Expertfit, o qual retornou uma função com diferentes parâmetros de entrada para cada processo, representando as distribuições de probabilidade que melhor descrevem cada um. No Erro! Fonte de referência não encontrada. encontram-se tais funções conforme suas representações descritas na Figura 14, configuradas para operar com minutos como unidade de tempo e escritas em unidades decimais inglesas devido ao idioma do software. 


\begin{tabular}{|c|c|c|}
\hline Processo & Tipo de distribuição & Representação no Flexsim HC \\
\hline Atendimento na Recepção & Erlang & erlang $(1.335046,1.633915,2.000000)$ \\
\hline Triagem hematológica & Beta & beta $(2.258635,5.245839,1.188355,1.905930)$ \\
\hline Triagem clínica & Log-normal & lognormal2 (1.972469, 1.635864, 0.950664) \\
\hline Recepção da ficha & Weibull & weibull $(0.211562,0.089494,2.000000)$ \\
\hline $\begin{array}{l}\text { Preparação da doação + doação* } \\
\text { (apenas doadores de sangue) }\end{array}$ & Pearson tipo $\mathrm{V}$ & pearsont5 $(6.167316,199.655179,9.470657)$ \\
\hline $\begin{array}{l}\text { Preparação da coleta + coleta de } \\
\text { sangue para doação de medula* } \\
\text { (apenas coleta de medula) }\end{array}$ & $8,35 * *$ & - \\
\hline $\begin{array}{l}\text { Preparação da doação + aférese* } \\
\text { (apenas doadores por aférese) }\end{array}$ & Beta & $\begin{array}{c}\text { beta }(65.641232,87.110996,0.455710 \text {, } \\
0.286805)\end{array}$ \\
\hline Hidratação & Beta & $\begin{array}{c}\text { beta }(3.049770,10.275460,0.972675 \\
1.071199)\end{array}$ \\
\hline \multicolumn{3}{|c|}{$\begin{array}{l}\text { *A preparação da doação não pode ser mensurada por não ser possível discerni-la do processo de doação ou } \\
\text { coleta. } \\
\text { **Houve apenas } 1 \text { doador em que foi possível coletar o tempo de coleta de sangue para doação de medula. }\end{array}$} \\
\hline
\end{tabular}

Figura 14 - Funções de distribuição de probabilidade dos processos.

Fonte: Autoria própria (2018).

Já para as frequências de chegada, foram coletados os intervalos de chegada durante os dias das filmagens, cujas distribuições correspondentes encontram-se na Erro! Fonte de referência não encontrada., também conforme descritas na Figura 15.

\begin{tabular}{|c|c|c|}
\hline Dia & Tipo de distribuição & Função de distribuição \\
\hline 1 & Beta & beta $(0.647171,36.195802,0.743956,2.349432)$ \\
\hline 2 & Beta & beta $(0.000000,75.198718,0.287442,1.077021)$ \\
\hline 3 & Johnson $S_{\mathrm{B}}$ limitada & johnsonbounded $(0.362301,91.524064,1.791949,0.754757)$ \\
\hline 4 & Johnson $S_{\mathrm{B}}$ limitada & johnsonbounded $(0.463062,208.700317,1.898834,0.677667)$ \\
\hline 5 & Pearson tipo VI & pearsont6( $0.000000,71.618097,0.565292,2.699300)$ \\
\hline 6 & Exponencial & exponential $(0.098562,7.606772)$ \\
\hline
\end{tabular}

Figura 15 - Funções de distribuição de probabilidade para a frequência de chegada. Fonte: Autoria própria (2018).

Utilizando-se a Eq. 01, foram calculados os números de replicações necessárias para cada dia a fim de se obter um valor de lead time com nível de confiança de $95 \%$ e erro de $5 \%$. O modelo foi então replicado para cada frequência de chegada e o número de candidatos a doação médio por dia após as replicações foi comparado com o número de candidatos a doação real daquela frequência de chegada a fim de validar o modelo. Os resultados encontram-se na

Tabela 1. 


\section{Tabela 1}

Número de replicações e candidatos a doação para cada frequência de chegada

\begin{tabular}{cccccc}
\hline Dia & $\begin{array}{c}\text { Número de } \\
\text { replicaçóes } \\
\text { necessárias }\end{array}$ & $\begin{array}{c}\text { Número de } \\
\text { replicações } \\
\text { realizadas }\end{array}$ & $\begin{array}{c}\text { Número de } \\
\text { candidatos a } \\
\text { doação real no } \\
\text { dia }\end{array}$ & $\begin{array}{c}\text { Número de } \\
\text { candidatos a } \\
\text { doação médio no } \\
\text { dia simulado }\end{array}$ & $\begin{array}{c}\text { Erro absoluto } \\
\text { entre o número } \\
\text { de candidatos a } \\
\text { doação }\end{array}$ \\
\hline 1 & 86 & 87 & 78 & 76 & 2 \\
2 & 78 & 83 & 41 & 45 & 4 \\
3 & 78 & 80 & 57 & 54 & 3 \\
4 & 65 & 67 & 28 & 30 & 4 \\
5 & 64 & 70 & 27 & 31 & 1 \\
6 & 94 & 100 & 51 & 50 & \\
\hline
\end{tabular}

Fonte: Autoria própria (2018).

O baixo valor do erro absoluto entre o número de candidatos a doação nas situações real e simulada demonstram haver correspondência entre os sistemas real e simulado, validando assim o modelo.

\subsection{Correlação entre lead time e número de candidatos a doação}

Após as replicações de cada dia, foram calculados os lead time médios de cada dia e seus respectivos desvios-padrões, bem como seus intervalos de confiança utilizando a Eq. 02, considerando um nível de confiança de $95 \%$ e um erro de 5\%, os quais são apresentados na

Tabela 2

Intervalos de confiança para cada dia

\begin{tabular}{cccccc}
\hline Dia & $\begin{array}{c}\text { Número de } \\
\text { candidatos a } \\
\text { doação médio } \\
\text { no dia simulado }\end{array}$ & $\begin{array}{c}\text { Lead time } \\
\text { médio simulado } \\
(\mathrm{min})\end{array}$ & Desvio Padrão & $\begin{array}{c}\text { Semi-intervalo } \\
\mathrm{h}(\mathrm{min})\end{array}$ & $\begin{array}{c}\text { Intervalo de } \\
\text { confiança (min) }\end{array}$ \\
\hline 1 & 76 & 53,75 & 12,25 & 2,62 & {$[51,13-56,37]$} \\
2 & 45 & 54,68 & 11,93 & 2,64 & {$[52,04-57,32]$} \\
3 & 54 & 52,79 & 10,94 & 2,45 & {$[50,34-55,24]$} \\
4 & 30 & 51,65 & 10,37 & 2,59 & {$[49,06-54,24]$} \\
5 & 31 & 52,71 & 10,45 & 2,55 & {$[50,16-55,26]$} \\
6 & 50 & 56,90 & 13,6 & 2,73 & {$[54,17-59,63]$} \\
\hline
\end{tabular}

Fonte: Autoria própria (2018).

Tabela 2

Intervalos de confiança para cada dia

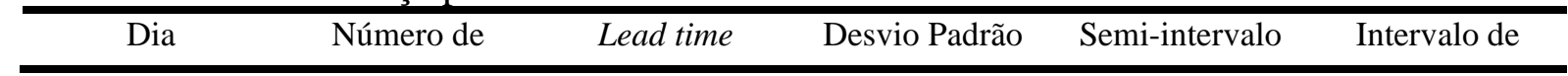




\begin{tabular}{cccccc}
\hline & $\begin{array}{c}\text { candidatos a } \\
\text { doação médio } \\
\text { no dia simulado }\end{array}$ & $\begin{array}{c}\text { médio simulado } \\
(\mathrm{min})\end{array}$ & & $\mathrm{h}(\mathrm{min})$ & confiança (min) \\
\hline 1 & 76 & 53,75 & 12,25 & 2,62 & {$[51,13-56,37]$} \\
2 & 45 & 54,68 & 11,93 & 2,64 & {$[52,04-57,32]$} \\
3 & 54 & 52,79 & 10,94 & 2,45 & {$[50,34-55,24]$} \\
4 & 30 & 51,65 & 10,37 & 2,59 & {$[49,06-54,24]$} \\
5 & 31 & 52,71 & 10,45 & 2,55 & {$[50,16-55,26]$} \\
6 & 50 & 56,90 & 13,6 & 2,73 & {$[54,17-59,63]$} \\
\hline
\end{tabular}

Fonte: Autoria própria (2018).

Para analisar a correlação entre as variáveis propostas, os pares de dados foram plotados em um gráfico de dispersão, conforme Figura 16.

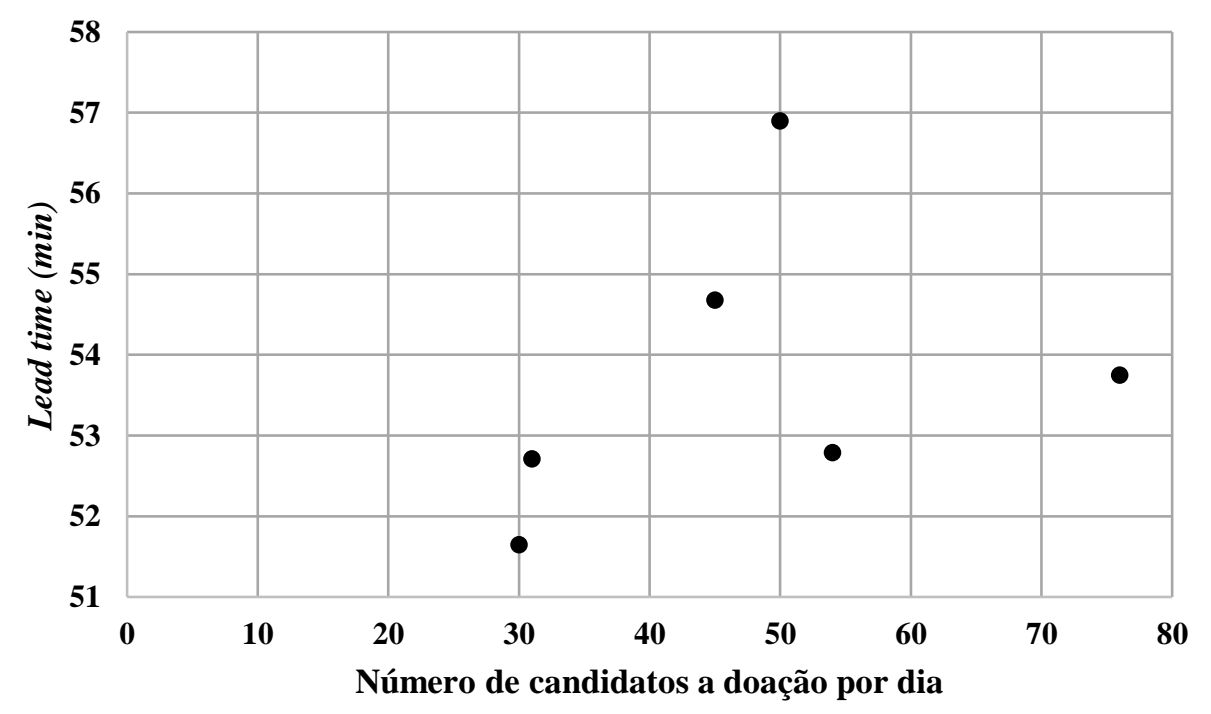

Figura 46 - Gráfico de dispersão para análise de correlação

Fonte: Autoria própria (2018).

Utilizando a Eq. 03, calculou-se o coeficiente de correlação entre as variáveis estudadas, obtendo um valor igual a 0,34. Assim, conforme explicado por Bussab e Morettin (2017), uma vez que o valor do coeficiente de correlação está muito próximo do zero, a correlação entre as variáveis lead time e número de candidatos a doação é nula no intervalo de dados analisado. Isso é evidenciado ao se comparar as variações dos valores da segunda e terceira coluna da

Tabela 2

Intervalos de confiança para cada dia

\begin{tabular}{cccccc}
\hline Dia & $\begin{array}{c}\text { Número de } \\
\text { candidatos a } \\
\text { doação médio } \\
\text { no dia simulado }\end{array}$ & $\begin{array}{c}\text { Lead time } \\
\text { médio simulado } \\
(\mathrm{min})\end{array}$ & Desvio Padrão & $\begin{array}{c}\text { Semi-intervalo } \\
\mathrm{h}(\mathrm{min})\end{array}$ & $\begin{array}{c}\text { Intervalo de } \\
\text { confiança (min) }\end{array}$ \\
\hline 1 & 76 & 53,75 & 12,25 & 2,62 & {$[51,13-56,37]$} \\
\hline
\end{tabular}




\begin{tabular}{llllll}
\hline 2 & 45 & 54,68 & 11,93 & 2,64 & {$[52,04-57,32]$} \\
3 & 54 & 52,79 & 10,94 & 2,45 & {$[50,34-55,24]$} \\
4 & 30 & 51,65 & 10,37 & 2,59 & {$[49,06-54,24]$} \\
5 & 31 & 52,71 & 10,45 & 2,55 & {$[50,16-55,26]$} \\
6 & 50 & 56,90 & 13,6 & 2,73 & {$[54,17-59,63]$} \\
\hline
\end{tabular}

Fonte: Autoria própria (2018).

, pois as variações percentuais do lead time médio não correspondem às variações do número de candidatos a doação, conforme ilustrado pela

Tabela 3, na qual está em ordem crescente do número de candidatos a doação médio.

\section{Tabela 3}

Variação do número de candidatos a doação médio e do lead time médio simulados

\begin{tabular}{cccc}
\hline \multicolumn{2}{c}{ Número de candidatos a doação médio no dia } & \multicolumn{2}{c}{ Lead time médio simulado } \\
simulado & $\begin{array}{c}\text { Variação percentual em } \\
\text { relação ao valor anterior }\end{array}$ & Valor (min) & $\begin{array}{c}\text { Variação percentual em } \\
\text { relação ao valor anterior }\end{array}$ \\
\hline Valor & - & 51,65 & - \\
& $3,33 \%$ & 52,71 & $2,1 \%$ \\
31 & $45,16 \%$ & 54,68 & $3,7 \%$ \\
45 & $11,11 \%$ & 56,90 & $4,1 \%$ \\
50 & $8,00 \%$ & 52,79 & $-7,2 \%$ \\
76 & $40,74 \%$ & 53,75 & $1,8 \%$ \\
\hline
\end{tabular}

Fonte: Autoria própria (2018).

É possível observar que, embora a quantidade de candidatos a doação sempre aumente

na

Tabela 3, em 4 dias observa-se um aumento do lead time médio porém, em 1 dia, há uma redução desse mesmo valor, evidenciando a falta de correlação entre as variáveis.

Essa falta de correlação pode ser consequência de diversos fatores, como: a influência que o tamanho das filas formadas podem exercer na velocidade de atendimento, como no estudo realizado por Oliveira, Neto e Cybis (2003), no qual foi evidenciado uma redução de 6,45\% nos tempos de atendimento de uma praça de pedágio em períodos de alto congestionamento, enquanto em períodos de baixo congestionamento observou-se um aumento de 13,21\% desse mesmo tempo; e o possível superdimensionamento da capacidade efetiva do HRM, projetada para atender uma demanda maior da que de fato ocorre. 


\section{CONCLUSÃO}

Por meio da utilização da simulação computacional e métodos estatísticos, foi possível avaliar a correlação entre o número de candidatos a doação em um dia de funcionamento com o lead time do doador de sangue no HRM.

Com o apoio do software Flexsim HC, foi possível construir o modelo que representasse a rotina do HRM, no qual foram incorporados os três tipos de processos realizados pela instituição: a doação de sangue, a doação por aférese e a coleta de sangue para doação de medula, bem como seus respectivos tempos de processos e intervalos de chegada.

Foram utilizados dados de 6 dias de operação, nos quais o número de candidatos a doação variou de 30 a 76 candidatos e, para cada respectivo dia, foram calculados os lead times médios em um intervalo de confiança com $95 \%$ de nível de confiança e 5\% de erro. A análise de correlação utilizando o método do coeficiente de correlação resultou num valor do coeficiente de 0,34, indicando não haver correlação entre as variáveis estudadas, conforme pode ser observado comparando as variações percentuais de cada variável para pares de dias.

Como propostas para estudos futuros, sugere-se analisar os possíveis fatores que podem estar causando essa ausência de correlação entre as variáveis estudadas, como a influência que o tamanho da fila de doadores pode estar exercendo na velocidade dos processos e o dimensionamento da capacidade efetiva do HRM.

\section{REFERÊNCIAS}

ABPM. Association of Business Process Management. (2013). Guia para o Gerenciamento de Processos de Negócio - Corpo Comum de Conhecimento, v. 3.

Alfonso, E., Xie, X., Augusto, V., \& Garraud, O. (2012). Modeling and simulation of blood collection systems. Health care management science, 15(1), 63-78. https://doi.org/10.1007/s10729-011-9181-8

Alfonso, E., Xie, X., Augusto, V., \& Garraud, O. (2013). Modelling and simulation of blood collection systems: improvement of human resources allocation for better costeffectiveness and reduction of candidate donor abandonment. Vox Sanguinis, 104(3), 225233. https://doi.org/10.1111/vox.12001

Alfonso, E., Xie, X., \& Augusto, V. (2015). A simulation-optimization approach for capacity planning and appointment scheduling of blood donors based on mathematical programming representation of event dynamics. In 2015 IEEE International Conference on Automation Science and Engineering (CASE) (pp. 728-733). IEEE. Disponível em: $<$ https://ieeexplore.ieee.org/stamp/stamp.jsp?tp=\&arnumber=7294167 >. Acesso em: 19 abr. 2018. 
Banks, J., \& Carson, J. S. (1984). Discrete-Event System Simulation. Englewood Cliffs: Prendice-Hall.

Barnes, R. M. (1977). Estudo de movimentos e de tempos: projeto e medida do trabalho. Editora Edgard Blucher.

Barrios, M. A. O., \& Jiménez, H. F. (2016). Use of six sigma methodology to reduce appointment lead-time in obstetrics outpatient department. Journal of medical systems, 40(10), 220.

Baş, S., Carello, G., Lanzarone, E., \& Yalçındağ, S. (2018). An appointment scheduling framework to balance the production of blood units from donation. European Journal of Operational Research, 265(3), 1124-1143. https://doi.org/10.1016/j.ejor.2017.08.054

Bateman, R., Bowden, R., Gogg, T., Harrel, C., Mott, J., \& Montevechi, J. (2013). Simulação de Sistemas - Aprimorando Processos de Logística, Serviços e Manufatura. Rio de Janeiro: Elsevier.

Beliën, J., \& Forcé, H. (2012). Supply chain management of blood products: A literature review. European Journal of Operational Research,217(1), 1-16. https://doi.org/10.1016/j.ejor.2011.05.026

Bertrand, J. W. M., \& Fransoo, J. C. (2016). Modelling and simulation. In Research methods for operations management (pp. 306-346). Routledge.

Bittar, O. J. N. V. (1999). Gestão de processos e certificação para qualidade em saúde. Revista da Associação Médica Brasileira, 45(4), 357-363.

Brasil. ANVISA. Agência Nacional de Vigilância Sanitária. Resolução - RDC nº 153, de 14 de junho de 2004. (2004). Regulamento Técnico para os procedimentos hemoterápicos, incluindo a coleta, o processamento, a testagem, o armazenamento, o transporte, o controle de qualidade e o uso humano de sangue, e seus componentes, obtidos do sangue venoso, do cordão umbilical, da placenta e da medula óssea. Alterada por Portaria $\mathrm{N}^{\mathrm{o}} 2.712$ de 12 de novembro de 2013. Diário Oficial da União $n^{\circ} 221$ - Ministério da Saúde. Brasília, DF, 13 Novembro 2013. Disponível em: <http://www.saude.mg.gov.br/images/documentos/RES_153.pdf.>. Acesso em: 19 abr. 2018.

Brasil. Portal Brasil. (2017). Apenas 1,8\% dos brasileiros doam sangue, saiba como se tornar um doador. Disponível em: <http://www.brasil.gov.br/saude/2017/06/apenas-1-8-dosbrasileiros-sao-doadores-de-sangue >. Acesso em: 18 abr. 2018.

Ministério da Saúde. Secretaria de Atenção à Saúde. Departamento de Atenção Especializada. (2010). Guia para o uso de hemocomponentes. Brasília: Editora do Ministério da Saúde.

Buhaug, H. (2002). Long waiting lists in Hospital. British Medical Journal, Macmillan Health Administration Press HSR.

Bussab, W. O., \& Morettin, P. A. (2017). Estatística básica. São Paulo: Saraiva.

Chung, R. D., Hunter-Smith, D. J., Spychal, R. T., Ramakrishnan, V. V., \& Rozen, W. M. (2017). A systematic review of intraoperative process mapping in surgery. Gland surgery, 6(6), 715. http://dx.doi.org/10.21037/gs.2017.11.02

Flexsim. (2018). Healthcare Simulation Software - Experience The Power Of Flexsim HC. Disponível em: <https://healthcare.flexsim.com/healthcare-simulation-software/>. Acesso em: 28 abr. 2018.

de Freitas Filho, P. J. (2001). Introdução à modelagem e simulação de sistemas: com aplicações em Arena. Visual Books.

Geronimo, B., \& Antonelli, G. (2017). Modelagem e Simulação Dinâmica do Banco de Sangue Maringá - Um Estudo de Caso. Ed. Novas Edições Acadêmicas. 
Graeml, A., \& Peinado, J. (2007). Administração da Produção: operações industriais e serviços. Curitiba: UnicenP.

Gurupur, V. P., Suh, S. C., Selvaggi, R. R., Karla, P. R., Nair, J. S., \& Ajit, S. (2012). An approach for building a personal health information system using conceptual domain knowledge. Journal of medical systems, 36(6), 3685-3693. https://doi.org/10.1007/s10916012-9842-2

Hemonorte. Hemocentro do Rio Grande do Norte Dalton Cunha. (2013). Tipos de Doação de Sangue. \&PAGE=0\&PARM=null\&LBL=Institui\%C3\%A7\%C3\%A3o >. Acesso em: 26 jun. 2018.

Hunt, V. D. (1996). Process mapping: how to reengeneer your business process.

INCA. Instituto Nacional de Câncer José Alencar Gomes da Silva. (2015). Como Se Tornar Um Doador. Disponível em: <http://redome.inca.gov.br/doador/como-se-tornar-umdoador/>. Acesso em: 27 jun. 2018.

Joukes, E., Abu-Hanna, A., Cornet, R., \& de Keizer, N. F. (2018). Time Spent on Dedicated Patient Care and Documentation Tasks Before and After the Introduction of a Structured and Standardized Electronic Health Record. Applied clinical informatics, 9(01), 046-053. https://doi.org/10.1055/s-0037-1615747

Kalman, H. K. (2002). Process mapping: Tools, techniques, \& critical success factors. Performance Improvement $\quad$ Quarterly, 15(4), http://doi.wiley.com/10.1111/j.1937-8327.2002.tb00265.x

Katsaliaki, K., \& Brailsford, S. C. (2007). Using simulation to improve the blood supply chain. Journal of the Operational Research Society, 58(2), 219-227. https://doi.org/10.1057/palgrave.jors.2602195

Lowalekar, H., \& Ravichandran, N. (2010). Model for blood collections management. Transfusion, 50(12pt2), 2778-2784. https://doi.org/10.1111/j.15372995.2010.02944.x

Martins, P., \& Laugeni, F. (2005). Administração da Produção (2a ed.). São Paulo: Saraiva.

Morabito, R., Pureza, V. (2018). Modelagem e Simulação. In Cauchick-Miguel, P. Metodologia de pesquisa em engenharia de produção e gestão de operações. ( $3^{a}$ Ed., Cap. 8, pp. 167-193). Rio de Janeiro: Elsevier.

Nguyen, T. B. T., Sivakumar, A. I., \& Graves, S. C. (2015). A network flow approach for tactical resource planning in outpatient clinics. Health care management science, 18(2), 124-136.

de Oliveira, M. L., Neto, J. M. R., \& Cybis, H. B. B. (2003). A Influencia do tamanho das filas na capacidade de Atendimento das praças de pedágio. In XVII Congresso de Pesquisa e Ensino em Transportes (pp. 1264-1275).

Ortiz, M. A., McClean, S., Nugent, C. D., \& Castillo, A. (2016, November). Reducing appointment lead-time in an outpatient department of gynecology and obstetrics through discrete-event simulation: a case study. In International Conference on Ubiquitous Computing and Ambient Intelligence (pp. 274-285). Springer, Cham.

Osorio, A. F., Brailsford, S. C., Smith, H. K., Forero-Matiz, S. P., \& Camacho-Rodríguez, B. A. (2017). Simulation-optimization model for production planning in the blood supply chain. Health care management science, 20(4), 548-564. https://doi.org/10.1007/s10729016-9370-6

Paraná. Secretaria da Saúde. (2013). Doação por Aférese. Disponível em: $<$ http://www.saude.pr.gov.br/modules/conteudo/conteudo.php?conteudo=2986>. Acesso em: 24 jun. 2018. 
Shannon, R. E. (1975). Systems Simulation: The Art and Science. Englewood Cliffs: Prendice-Hall.

Silva Júnior, J. B., Costa, C. D. S., \& Baccara, J. P. D. A. (2015). Regulação de sangue no Brasil: contextualização para o aperfeiçoamento. Revista Panamericana de Salud Pública, 38, 333-338.

Singh, S., Upadhyaya, S., Deshmukh, P., Dongre, A., Dwivedi, N., Dey, D., \& Kumar, V. (2018). Time motion study using mixed methods to assess service delivery by frontline health workers from South India: methods. Human resources for health, 16(1), 17. https://doi.org/10.1186/s12960-018-0279-7

Takagi, H., Kanai, Y., \& Misue, K. (2017). Queueing network model for obstetric patient flow in a hospital. Health care management science, 20(3), 433-451. https://doi.org/10.1007/s10729-016-9363-5

Testik, M. C., Ozkaya, B. Y., Aksu, S., \& Ozcebe, O. I. (2012). Discovering blood donor arrival patterns using data mining: A method to investigate service quality at blood centers. Journal of medical systems, 36(2), 579-594. https://doi.org/10.1007/s10916-0109519-7

Tubino, D. F. (2007). Planejamento e Controle da Produção. São Paulo: Atlas.

Vaccari, M., Montasser, W., Tudor, T., \& Leone, L. (2017). Environmental audits and process flow mapping to assess management of solid waste and wastewater from a healthcare facility: an Italian case study. Environmental monitoring and assessment, 189(5), 239. https://dx.doi.org/10.1007/s10661-017-5940-4 